\title{
De Garengeot hernia - Uncommon cause of a right groin pain
}

\author{
Maximilian Anheier ${ }^{*}$ and KH Schultheis ${ }^{2}$ \\ ${ }^{1}$ Senior Physician of the Department of General, Visceral and Thoracic Surgery, Protestant Hospital Bethesda Mönchengladbach Ludwig-Weber-Straße 15, 41061 \\ Mönchengladbach, Germany \\ ${ }^{2}$ Department of General, Visceral, Thoracic and Endocrine Surgery, Augusta Hospital Dusseldorf, Amalienstraße 9, 40472, Dusseldorf, Germany
}

\begin{abstract}
De Garengeot hernia is described as the appendix vermiformis in a femoral hernia sac. It is a very rare disease with difficulties in diagnosis and therapy. Most patients present with a painful swelling in the right groin. Open and laparoscopic surgery is described. We present the case of a de Garengeot hernia and laparoscopic appendectomy with simultaneous transabdominal preperitoneal hernia repair (TAPP) and a review of the literature.
\end{abstract}

\section{Introduction}

De Garengeot hernia is described as the presence of the appendix within a femoral hernia. It was first described by French surgeon Rene Jaques de Garengeot in 1731. Incidence is reported with $0,8-1 \%$, simultaneous acute appendicitis with $0,08-0,5 \%$ [1]. Since 1960 less than 100 cases are reported [2]. Diagnosis of a de Garengeot hernia is difficult. Most patients present with a painful swelling in the right groin [5]. Laboratory investigation shows most often leukocytosis. Ultrasound and CT investigation can show a strangulated femoral hernia [7]. Typical features of acute appendicitis are not often present and diagnosis is often made intraoperatively [9]. The treatment of a de Garengeot hernia is emergency surgery. Open appendectomy and hernia repair with or without mesh and also laparoscopic approaches are described. Because de Garengeot hernia is a rare entity there is no standard treatment. We report the case of a 68 -year-old woman with de Garengeot hernia and laparoscopic appendectomy with simultaneous transabdominal preperitoneal hernia repair (TAPP) and a review of the literature.

\section{Case report}

A 68-year-old woman was send to our surgical department with a swelling in the right groin for 3 days. Her previous medical history includes only hypertension and no operation. There was no vomiting or emesis. In the clinical examination of the patient there was a swelling in the right groin with tenderness on palpation. The abdomen was inconspicuous. Blood investigation shows regular account of white blood cells and CRP. Ultrasound of the abdomen and the right groin showed an unclear tubular structure in a femoral hernia. Further CTscan also showed a tubular structure in the right femoral hernia and strangulation of the right ovary inside femoral hernia was the first diagnosis (Figure 1). Patient was transferred to operation theatre for laparoscopic exploration.

Intraoperatively the diagnosis de Garengeot hernia with incarceration of the appendix in the femoral hernia sac was made (Figure 2). The appendix was removed without problems to the

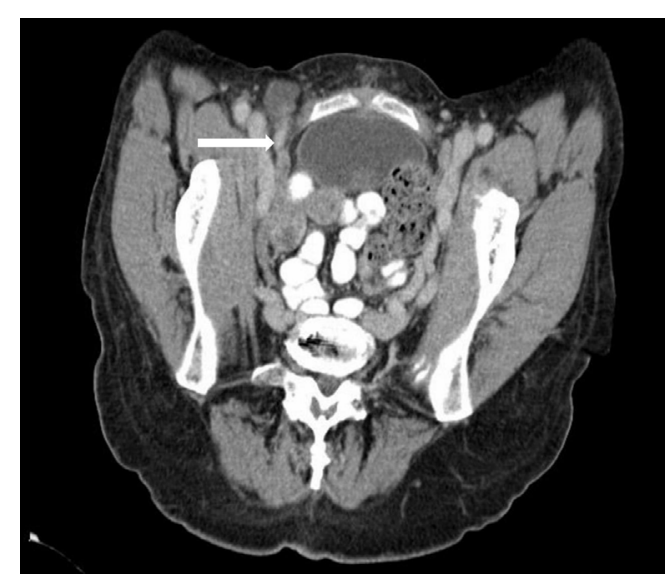

Figure 1. CT-scan with tubular structure in a femoral hernia

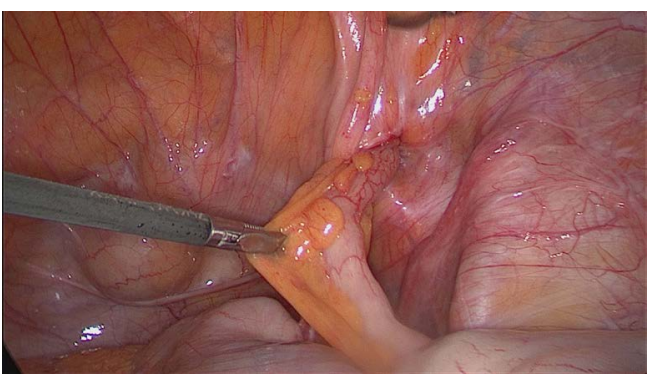

Figure 2. The incarcerated appendix in the femoral hernia

${ }^{\star}$ Correspondence to: Maximilian Anheier, Senior Physician of the Department of General, Visceral and Thoracic Surgery, Protestant Hospital Bethesda Mönchengladbach Ludwig-Weber-Straße 15, 41061 Mönchengladbach, Germany, Tel: $02161981-2250$; Fax: 02161 981-2260; E-mail: Maximilian.Anheier@ mg.johanniter-kliniken.de

Received: May 21, 2018; Accepted: May 29, 2018; Published: June 04, 2018 
abdomen and laparoscopic appendectomy was performed. A stapler was used for the appendectomy and additional sutures were made. Because appendicitis was mild and there were no signs of peritonitis of fluids inside the abdominal cavity we decided to perform hernia repair with a mesh. Therefor the peritoneum was incised, the PolypropylenePoliglecapron mesh (size $15 \times 15 \mathrm{~cm}$, Ultrapro@) was placed in the preperitoneal cavity and peritoneum was closed with sutures afterwards. Preoperative the patient received a single-shot therapy with cefuroxim. The patient recovered fast from our operation and was dismissed at the $3^{\text {rd }}$ day from our hospital.

\section{Discussion}

The finding of an appendix in a femoral hernia sac is a rare entity which is often diagnosed intraoperatively. It is important to differ between de Garengeot hernia and Amyand hernia which is characterized by a vermiform appendix in an inguinal hernia sac. The incidence of this also rare entity is $1 \%[3,4]$. Amyand hernia is more often reported in men whereas de Garengeot hernia is more often observed in postmenopausal women [5]. Femoral hernia has a higher risk of incareation (5-20\%) because of the narrow and rigid ring [6]. Because of the anatomy of the vermiform appendix de Garengeot hernia is more often on the right side. It is uncertain whether the incarceration of the appendix in tight ring of the femoral hernia leads to appendicitis or appendicitis comes first and afterwards it comes to a migration to the femoral hernia $[8,10]$. Therefore, it is discussed that appendicitis in a femoral hernia could prevent the abdominal cavity from infection and because of this fact the clinical findings are not obvious for appendicitis [11].

Diagnosis of a de Garengeot hernia is difficult. Most patients present with a painful swelling in the right groin. Other reported symptoms are bowel symptoms such as nausea or vomiting. Duration of the symptoms can extremely differ. There are reported cases with a duration of symptoms of months [17]. Laboratory investigation shows leukocytosis in $67 \%$ and an elevated level of CRP in $83 \%$ [5]. Also, in $\mathrm{CT}$ and ultrasound the diagnosis is challenging with better findings in CT diagnosis. Ultrasound can reveal bowel contents in the hernia sac while CT scan can show a low-lying caecum with a tubular structure inside the femoral hernia. CT-scan can also show intramural air density in an incarcerated hernia sac indicating intestinal involvement. CT scan has a $98 \%$ sensitivity and specify for diagnosing or excluding appendicitis within a hernia sac $[7,12,13]$.

Because de Garengeot-hernia is a rare entity there is no standard treatment. Therapy includes appendectomy and hernia repair with or without mesh. Open and laparoscopic procedures are described whereupon the laparoscopic way is still controversial [14].

Most reported procedures are open femoral hernia repair und appendectomy through the peritoneal sac. The classically approaches are Lockwood's infra-inguinal, Lotheissen's trans-inguinal and McEvedy's high approach [15]. A laparoscopic appendectomy followed by totally extraperitoneal femoral hernia repair (TEP) was first described in 2013 [16]. Laparoscopic appendectomy followed by transabdominal preperitoneal hernia repair (TAPP) as we performed is also described in several cases $[18,19]$. The choice of treatment depends on the surgeon and the performance of the patient.

In our presented case the exact diagnosis was made intraoperatively as in the most reported cases [20]. We decided to do laparoscopy under the diagnosis of an incarcerated ovary or an incarcerated fallopian tube in a femoral hernia. Therefore, laparoscopic repair is described by Soeta et al. [21]. Intraoperatively we made the diagnosis of an incarcerated vermiform appendix in a right femoral hernia. Because appendicitis was mild and because there were no signs of peritonitis we decided to do laparoscopic appendectomy following transabdominal preperitoneal hernia repair (TAPP). Because the peritoneum is closed with a suture after the mesh is placed in the preperitoneal space there is no contact between the mesh and the abdominal cavity. Therefore, this approach seems a good technique also in presence of appendicitis.

Because preoperative diagnosis of de Garengeot hernia is difficult we recommend doing diagnostic laparoscopy in case of nonspecific abdominal pain and suspicion of appendicitis or incarcerated femoral or inguinal hernia. In case of a de Garengeot hernia and in absence of a perforation of the appendix laparoscopic appendectomy followed by transabdominal preperitoneal hernia repair with a mesh is possible. This procedure includes the benefit of laparoscopic operations as fast recovery and small incisions. In case of peritonitis laparoscopic appendectomy is also possible with hernia repair afterwards or with an additional open inguinal approach.

\section{Conclusion}

Incarcerated femoral hernia can obtain the vermiform appendix.

We recommend laparoscopic appendectomy followed by transabdominal preperitoneal hernia repair (TAPP) with a mesh in de Garengeot hernia in absence of peritoneal abscess.

\section{References}

1. Akbari K, Wood C, Hammad A, Middleton S (2014) De Garengeot's hernia: Our experience of three cases and literature review. BMJ Case Rep. [Crossref]

2. Mizumoto R, Hendahewa R, Premaratne G (2016) De Garengeot hernia-use of a novel surgical approach and literature review. Int J Surg Case Rep 19: 127-130. [Crossref]

3. Kagan Coskun A, Kilbas Z, Yigit T, Simsek A, Harlak A (2012) De Garengeot's hernia: The importance of early diagnosis and its complications. Hernia 16: 731-733. [Crossref]

4. Pan CW, Tsao MJ, Su MS (2015) A case of De Garengeot hernia requiring early surgery. BMJ Case Rep. [Crossref]

5. Michalinos A, Moris D, Vernadakis S (2014) Amyand's hernia: A review. Am J Surg 207: 989-995. [Crossref]

6. Bidarmaghz B, Tee CL (2017) A case of De Garengeot hernia and literature review. BMJ Case Rep. [Crossref]

7. Kromka W, Rau AS, Fox CJ (2018) Amyand's hernia with acute gangrenous appendicitis and cecal perforation: A case report and review of the literature. Int J Surg Case Rep 44: 8-10. [Crossref]

8. Erdas E, Sias L, Licheri S, Secci L, Aresu S, et al. (2013) De Garengeot hernia with acute appendicitis. G Chir 34: 86-89. [Crossref]

9. Kalles V, Mekras A, Mekras D, Papapanagiotou I, Al-Harethee W, et al. (2013) De Garengeot's hernia: A comprehensive review. Hernia 17: 177-182. [Crossref]

10. Bloom A, Baio FE, Kim K, Fernandez-Moure JS, Reader M (2017) Diagnosis and operative management of a perforated de Garengeot hernia. Int $J$ Surg Case Rep 41: 114-116. [Crossref]

11. [Crossref] D'Ambrosio N, Katz D, Hines J (2006) Perforated appendix within a femoral hernia. AJR Am J Roentgenol 186: 906-907.

12. Maizlin ZV, Mason AC, Brown C, Brown JA (2007) CT findings of normal and inflamed appendix in groin hernia. Emerg Radiol 14: 97-100. [Crossref]

13. A Subaie S, Mustafa H, Al-Sharqawi N, Al-Haddad M, Feras Othman (2015) A case of de Garengeot hernia: the feasibility of laparoscopic transabdomnial preperitoneal hernia repair. Int J Surg Case Rep 16: 73-76. [Crossref]

14. Sorelli PG, El-Masry NS, Garrett WV (2009) Open femoral hernia repair: one skin incision for all. World J Emerg Surg 4: 44. [Crossref]

15. Beysens M, Haeck L, Vindevoghel K (2013) Laparoscopic appendectomy combined with TEP for der Garengeot hernia: case report. Acta Chir Belg 113: 468-470. [Crossref] 
16. Voitk AJ, MacFarlane JK, Estrada RL (1974) Ruptured appendicitis in femoral hernias: Report of two cases and review of the literature. Ann Surg 179: 24-26. [Crossref]

17. Ebisawa K, Yamazaki S, Kimura Y, Kashio M, Kurito K, et al. (2009) Acute appendicitis in an incarcerated femoral hernia: A case of De Garengeot hernia. Case Rep Gastroenterol 3: 313-317. [Crossref]

18. Comman A, Gaetzschmann P, Hanner T, Behrend M (2007) DeGarengeot hernia: transabdominal preperitoneal hernia repair and appendectomy. JSLS 11: 496-501. [Crossref]
19. Al-Subaie S, Mustafa H, Al-Sharqawi N, Al-Haddad M, Othman F (2015) A case of de Garengeot hernia: the feasibility of laparoscopic transabdominal preperitoneal hernia repair. Int J Surg Case Rep 16: 73-76. [Crossref]

20. Akopian G, Alexander M (2005) De Garengeot hernia: Appendicitis within a femoral hernia. Am Surg 71: 526-527. [Crossref]

21. Soeta N, Saito T, Nemoto T, Oshibe I, Gotoh M (2016) Laparoscopic repair of irreducible femoral hernia containing the fallopian tube alone: A case report. Surg Case Rep 2: 57. [Crossref]

Copyright: (C2018 Anheier M. This is an open-access article distributed under the terms of the Creative Commons Attribution License, which permits unrestricted use, distribution, and reproduction in any medium, provided the original author and source are credited. 